\title{
Bedtime procrastination: A self-regulation perspective on sleep insufficiency in the general population
}

Journal of Health Psychology 2016, Vol. 21 (5) 853-862 (C) The Author(s) 2014

Reprints and permissions: sagepub.co.uk/journalsPermissions.nav DOI: $10.1177 / 1359105314540014$ hpq.sagepub.com

@SAGE

\author{
Floor M Kroese, Catharine Evers, Marieke A \\ Adriaanse and Denise TD de Ridder
}

\begin{abstract}
Getting insufficient sleep has serious consequences in terms of mental and physical health. The current study is the first to approach insufficient sleep from a self-regulation perspective by investigating the phenomenon of bedtime procrastination: going to bed later than intended, without having external reasons for doing so. Data from a representative sample of Dutch adults $(N=243 \mathrm{I})$ revealed that a large proportion of the general population experiences getting insufficient sleep and regularly goes to bed later than they would like to. Most importantly, a relationship between self-regulation and experienced insufficient sleep was found, which was mediated by bedtime procrastination.
\end{abstract}

\section{Keywords}

community health psychology, health, health behavior, lifestyle, psychology, well-being

Insufficient sleep is known to have serious consequences in terms of mental and physical health. For example, research shows that a lack of sleep is related to concentration and memory problems (e.g. Ram et al., 2010), cardiovascular diseases and obesity (e.g. Buxton and Marcelli, 2010). Additionally, it has been found that the number of sleeping hours in the general population has decreased over the past decades (e.g. National Sleep Foundation, 2005). Although much research has been concerned with sleep insufficiency due to sleeping disorders (e.g. Swanson et al., 2011) or external factors (e.g. working nightshifts, Ohayon et al., 2010), we propose that insufficient sleep in the general population can be regarded as a typical health behavior problem where intentions to go to bed are not always in line with actual behavior.
The current aim is to introduce a novel selfregulation perspective on sleep insufficiency in the general population by employing a largescale prospective study. In addition to studies focusing on sleep disorders or external factors that may cause a lack of sleep, we approach the issue in terms of a bedtime procrastination problem.

The current study focuses on the intuitivebut surprisingly understudied - notion that people simply go to bed too late. More specifically, we investigate the phenomenon that people go

\footnotetext{
Utrecht University, The Netherlands

Corresponding author:

Floor M Kroese, Department of Clinical and Health

Psychology, Utrecht University, P.O. Box 80 I40, 3508 TC

Utrecht, The Netherlands.

Email: F.M.Kroese@uu.nl
} 
to bed later than they initially wanted to-an experience that receives much anecdotal but as of yet no empirical evidence - and that this is a matter of poor self-regulation. Going to bed later than intended, without having external reasons for doing so, is labeled bedtime procrastination. Procrastination is considered a demonstration of having poor self-regulation skills, as it reflects a typical intention-behavior gap (Steel, 2007).

Self-regulation refers to the "efforts undertaken by humans to alter their thoughts, feelings, desires and actions in the perspective of personally relevant goals" (De Ridder and De Wit, 2006: 2). In line with findings that people with low self-regulation skills are, for example, less well able to resist temptations and are typical procrastinators with academic tasks (Tangney et al., 2004), we propose that they are also more likely to postpone going to bed, despite their good intentions. Indeed, some first indications for the association between trait self-regulation and bedtime procrastination have been found (Kroese et al., 2014). The current study is the first to investigate bedtime procrastination in a large community sample and to include a prospective bedtime diary.

Approaching insufficient sleep for the first time from a self-regulation perspective has important implications. Theoretically, considering going to bed late as a self-regulation problem aligns this behavior with other health behaviors such as failing to adhere to a medical regime or eating too few fruits and vegetables. This means that theoretical models used to explain these behaviors may also be applicable to insufficient sleep. In addition, the current research may shed new light on earlier findings reported in the literature. Interesting in this regard are previously reported associations between getting too little sleep and lifestyle factors such as smoking, a lack of exercise, and obesity (Ohida et al., 2001; Strine and Chapman, 2005). Although it has been inferred that having an unhealthy lifestyle puts people at risk for sleeping problems (and, hence, improving a healthy lifestyle would facilitate sleep behaviors; Ohida et al., 2001), our current perspective suggests that insufficient sleep and other unhealthy lifestyle behaviors may all be associated to the same underlying issue of having low self-regulation skills. Finally, touching upon practical implications, the self-regulation perspective opens up new pathways to improve behavior, learning from strategies that are known to be effective for other self-regulation problems.

In sum, the present study will contribute to the literature in two ways: First, based on data from a large community sample, we will shed light on the prevalence of experienced sleep insufficiency in the general population. Second, we will be the first to investigate the association between self-regulation, bedtime procrastination and sleep insufficiency, highlighting a novel approach to the well-known problem of insufficient sleep. To this end, the current study employs a prospective design including baseline surveys and a bedtime diary taken 1 month later from a large adult community sample.

\section{Method}

\section{Participants}

Participants were members of the LISS (Longitudinal Internet Studies for the Social Sciences) panel, which is a representative sample of Dutch individuals who participate in monthly Internet surveys. The panel (administered by CentERdata, Tilburg University, The Netherlands) is based on a true probability sample of households drawn from the population register. Individuals who could not otherwise participate are provided with a computer and Internet connection. A total of 2637 participants filled out the baseline questionnaire and 2106 also completed the 7-day bedtime diary. The descriptive analyses were conducted for the baseline sample, excluding participants who indicated to work nightshifts or to have had treatment for sleeping problems (two largely overlapping categories; $N=206$ ), as these factors may have particular impact on sleep insufficiency as well. The remaining sample consisted of $54.5 \%$ females and participants on 
average were 50.7 years of age $(S D=18.1$, range 16-93). The majority of the sample lived together with a partner, either with $(35.6 \%)$ or without (39.2\%) children. A further $18.4 \%$ lived alone, and 5.4\% lived with (a) child(ren) but no partner; $1.3 \%$ had other living arrangements. Education levels were evenly distributed, with $36.3 \%$ having completed lower education (elementary school and/or lower secondary education), 33.1\% having medium-level education (vocational education and/or higher secondary education), and 30.2\% completed higher education (college and/or university); for $0.4 \%$ of participants the education level was missing.

\section{Procedure}

The survey and diary were distributed through the online system of the LISS panel. The baseline survey included questions on experienced sleep insufficiency, self-regulation, general procrastination, and bedtime procrastination. Demographics were collected from the LISS database that is updated yearly. The diary was assessed 1 month later and consisted of questionnaires regarding actual sleeping behavior (see Measures) that were assessed at seven consecutive days. CentERdata abides by the Dutch "protection of personal data act," which is derived from and consistent with European law (Directive 95/46/EC). Additionally, the current research proposal was reviewed by an internal review board prior to including the survey in the monthly panel questionnaires.

\section{Measures}

\section{Baseline}

Demographics and exclusion criteria. Demographics were retrieved from the LISS database and included gender, age, marital status, household composition, number of children living at home, employment status, education level, and ethnicity. In addition, participants were asked whether they "had ever received treatment for sleeping problems from a physician or therapist," and whether they worked nightshifts. Both questions had dichotomous response options (yes/no), and people who responded "yes" to one or both of these questions were excluded from the analyses (see the "Participants" section).

Self-control. Trait self-control is generally considered the main indicator of self-regulation skills on an individual differences level. It was assessed with the brief Self-Control Scale (Tangney et al., 2004; Cronbach's alpha = .79). The scale consists of 13 items (e.g. "I can resist temptations") that were answered on a scale from 1 (not at all like me) to 5 (very much like $m e$ ). Being widely used in self-control research, it has been shown to be associated with a range of behaviors such as work performance, weight control, and interpersonal functioning (De Ridder et al., 2012).

Bedtime procrastination. Bedtime procrastination was assessed with a 9-item scale that was created by the authors (Cronbach's alpha $=.88$, Appendix 1). Sample items are "I go to bed later than I would like to," and "I find it easy to quit doing other activities in the evening when it is time to go to bed" (reverse coded). Questions were answered on a scale from 1 (almost never) to 5 (almost always). The scale was assessed at baseline and at the start of the sleep diary 1 month later and showed good test-retest reliability (Pearson's $r=.79$ ).

Sleep descriptives. Participants were asked to indicate, on average, how many hours they typically sleep during week nights and weekend nights. Furthermore, they were asked to what extent they found this sufficient ("to what extent do you feel the number of hours of sleep you get is sufficient"), on a scale ranging from 1 (not at all) to 5 (largely so).

Indicators of insufficient sleep. A number of indicators of sleep insufficiency were assessed: experiencing insufficient sleep ("in an average week, how many days do you feel you had too little sleep"), going to bed late ("in an average week, how many days do you go to bed later than you would like to," and daytime fatigue ("in an 
average week, how many days do you feel tired during the day") were all answered in number of days (0-7). In addition, if participants provided an answer of 1 or more, they were asked to what extent they found this problematic (e.g. "to what extent do you find it problematic that you go to bed later than you would like to on [x] days of the week?"), on a scale from 1 (not at all) to 5 (very much).

External reasons. Participants were asked "to what extent is your bedtime generally affected by external circumstances that are outside of your control," to which they could respond on a scale from 1 (never) to 5 (always).

\section{Sleep diary}

Discrepancy planned-actual bedtimes. Each day, participants reported on the previous night and indicated at what time they wanted to go to bed, and at what time they actually went to bed. In addition, they could indicate whether there was any specific external reason (yes/no) that delayed their bedtime outside of their control. If so, participants were asked to describe the reason in an open-ended question. Provided reasons were later checked by the experimenter to decide whether they indeed presented factors that were outside a person's control (e.g. crying baby, sick husband, staying up for a daughter who came home late) or not (e.g. an interesting TV show). The difference between planned and actual bedtimes was calculated for each day. Disregarding days on which a valid external reason was provided, a mean discrepancy was calculated for the entire week.

Diary hours of sleep. Each day, participants reported how many hours they slept during the previous night (open-ended question).

Activities. For each day, participants reported what they were doing directly prior to going to bed. They could select several response options (i.e. watching TV, using the computer, doing household or administrative chores, activities outside of the house, social activities at home). An "other" option was also available, which they were asked to specify. This measure was used for descriptive analyses, in order to gain insight into the situations in which bedtime procrastination is most likely to occur.

\section{Results}

\section{Descriptive statistics}

In the baseline questionnaire, participants on average reported to sleep 7.2 hours $(S D=1.3)$ on week nights, which is a little less than the average hours of sleep calculated from the diaries $(M=7.4, S D=1.0)$. For weekend nights, baseline reports yielded an average of 7.9 hours $(S D=1.6)$, which was close to the average based on the diary $(M=7.8, S D=1.3)$. Almost $58 \%$ of the sample felt the amount of sleep they got on week nights was generally sufficient (scoring 4 or 5 on a 5-point scale), while $17.5 \%$ did not think so (scoring 1 or 2 ) and $24.6 \%$ was mixed or neutral (scoring 3 on this scale).

Frequencies of the indicators of insufficient sleep are reported in Table 1. It shows that over $50 \%$ of the sample reported feeling to have insufficient sleep on average on 2 or more days during the week and a roughly similar percentage $(53.1 \%)$ reported going to bed later than they want to on 2 or more days per week. Accordingly, almost $45 \%$ of the sample felt tired during the day on 2 or more days per week. Among those who reported experiencing insufficient sleep on one or more days, $78.5 \%$ found this at least somewhat problematic. Moreover, among people who indicated to go to bed later than they want to on one or more days, $70.6 \%$ found this at least somewhat problematic.

Regarding activities performed prior to going to bed, watching TV was most frequently selected (frequencies ranging from $55.3 \%$ on Saturday to $65.5 \%$ on Monday night), followed by using the computer (frequencies ranging from $11.0 \%$ on Saturday to $18.8 \%$ on Tuesday), social activities at home $(12.8 \%$ on Wednesday through $20.8 \%$ on Saturday), or "other" (13.3$16.0 \%)$. Household chores $(4.3-6.9 \%)$ and 
Table I. Frequencies of insufficient sleep indicators.

\begin{tabular}{llllll}
\hline How many days, in an average week ... & $0-1$ days & $2-3$ days & $4-5$ days & $6-7$ days & Mean (SD) \\
\hline $\begin{array}{l}\text {.. do you feel you have had too little } \\
\text { sleep }\end{array}$ & $45.5 \%$ & $31.4 \%$ & $15.8 \%$ & $7.3 \%$ & $2.1(2.0)$ \\
$\begin{array}{l}\text {.. do you go to bed later than you } \\
\text { would like to }\end{array}$ & $46.9 \%$ & $29.1 \%$ & $15.5 \%$ & $8.6 \%$ & $2.2(2.0)$ \\
\begin{tabular}{l}
.. do you feel tired during the day \\
\hline
\end{tabular} & $55.8 \%$ & $24.3 \%$ & $11.0 \%$ & $9.0 \%$ & $1.9(2.1)$ \\
\hline
\end{tabular}

Note: Participants responded by indicating any number between 0 and 7; the categories in the current table were only created for the ease of interpretation.

Table 2. Correlations.

\begin{tabular}{llllllll}
\hline & $\mathrm{I}$ & 2 & 3 & 4 & 5 & 6 & $M(S D)$ \\
\hline I. Bedtime procrastination & - & & & & & & $2.7(0.8)$ \\
2. Self-control & $-.39^{* *}$ & - & & & & & $3.4(0.6)$ \\
3. Insufficient sleep (days) & $.49^{* *}$ & $-.28^{* *}$ & - & & & & $2.1(2.0)$ \\
4. Fatigue (days) & $.28^{* *}$ & $-.25^{* *}$ & $.56^{* *}$ & - & & & $1.9(2.1)$ \\
5. Hours of sleep (baseline survey) & $-.24^{* *}$ & $.08^{* *}$ & $-.35^{* *}$ & $-.14^{* *}$ & - & & $7.2(1.3)$ \\
6. Hours of sleep (diary) & $-.17^{* *}$ & $.05^{*}$ & $-.30^{* *}$ & $-.10^{* *}$ & $.49^{* *}$ & - & $7.4(1.0)$ \\
7. Satisfaction w/hours of sleep & $-.43^{* *}$ & $.25^{* *}$ & $-.66^{* *}$ & $-.43^{* *}$ & $.43^{* *}$ & $.32^{* *}$ & $3.6(1.1)$ \\
\hline
\end{tabular}

$* p<.05 ; * * p<.001$.

activities outside one's own house $(2.2-8.9 \%)$ were selected least often. "Other" options that people mentioned were mostly reading, listening to music, or other hobbies (playing card games, knitting, etc.).

Correlations between various sleep outcomes and self-regulation variables (self-control, general procrastination, bedtime procrastination) are reported in Table 2 .

Notably, correlations between hours of sleep as reported at baseline and as calculated from the diaries were only moderate $(r=.49$ and .37 for hours of sleep on weekdays and weekends, respectively; $p<.001)$. In the remaining analyses, hours of sleep as calculated from the diaries will be used, as they were deemed more accurate than the baseline measure. Furthermore, the analyses include the average hours of sleep for weekdays only (i.e. Sunday through Thursday night) as weekend nights may include more atypical sleeping patterns. However, including average hours of sleep for the entire week yielded very similar results.

\section{Main analyses}

The purpose of the main analyses was to investigate the relationship between self-control and sleep outcomes (experienced insufficient sleep, reported hours of sleep in the diary, discrepancy between intended and actual bedtimes), and to test whether this association was mediated by bedtime procrastination. A series of hierarchical multiple regression analyses were performed, in which Step 1 always consisted of demographics (gender, age, living with a partner, number of children living at home, working, and studying), and Step 2 included generally experienced external reasons. The variables of interest (i.e. self-control and bedtime procrastination) were included in Steps 3 and 4, respectively. For the purpose of the current study, household composition was dichotomized into living with a partner versus living alone; the number of children living at home was considered as a separate factor. Furthermore, employment status was dummy coded into working or not working, and studying or not studying. 
Examining experienced insufficient sleep as the dependent variable, the analysis showed that each subsequent step significantly improved the model. Demographics in Step 1 explained 8.0\% of the variance, with $\operatorname{sex}(\beta=.08, p<.001)$ and age $(\beta=-.26, \mathrm{p}<.001)$ being significant predictors, while the number of children living at home $(\beta=.05, p=.06)$ and going to school $(\beta=-.05$, $p=.09)$ were marginally significant. Adding external reasons in Step 2 significantly improved the model; $F_{\text {change }}(1,2414)=125.8, p<.001$, and explained an additional $4.6 \%$ of the variance in experienced insufficient sleep. Indeed, generally experienced external reasons were a significant predictor $(\beta=.23, p<.001)$. The addition of self-control again significantly improved the model, $F_{\text {change }}(1,2414)=117.2$, $p<.001$, and added $4.0 \%$ of explained variance. Confirming our expectations, self-control $(\beta=-.21, p<.001)$ was indeed negatively associated with experiencing insufficient sleep. When bedtime procrastination was added to the model, the effect of self-control was reduced. Step 4 explained an additional $12.7 \%$ of the variance, $F_{\text {change }}(1,2412)=433.4, p<.001$, leading to a total explained variance of $29.3 \%$ for the entire model. Bedtime procrastination was the strongest predictor $(\beta=.40, p<.001)$, and selfcontrol $(\beta=-.08, p<.001)$, external reasons $(\beta=.15, p<.001)$, going to school $(\beta=-.07$, $p=.01)$, sex $(\beta=.08, p<.001)$, and age $(\beta=-.10, p<.001)$ also remained significant. More bedtime procrastination, lower self-control, more external reasons, not being a student, being female, and being younger were associated with greater experienced insufficient sleep. As the reduction of the effect of self-control upon introduction of bedtime procrastination to the model suggested possible mediation, bootstrapping analyses were performed. The analysis $(z=10,000)$ indeed confirmed a significant indirect effect of self-control on experienced insufficient sleep through bedtime procrastination $(95 \%$ CI of standardized indirect effect: $[-0.19,-0.15])$. Thus, the effect of self-control on insufficient sleep was partially mediated by bedtime procrastination. The relations are illustrated in Figure 1.
The analyses were repeated with number of hours of sleep and discrepancy between planned and actual bedtimes as dependent variables, yielding similar results. The final models of these analyses are presented in Table 3. Again, bootstrapping analyses confirmed significant indirect effects of self-control, through bedtime procrastination, on hours of sleep and discrepancy between planned and actual bedtimes (95\% CI: [0.05, 0.09] and [-0.12, -0.08$]$, respectively).

\section{General discussion}

It is clear from the literature that getting sufficient sleep is crucial for human health and wellbeing. In that light, it is surprising to see that prior research on sleeping behavior has largely focused on specific subsamples such as sleep disorder patients, nightshift workers, or children, while far less is known about insufficient sleep among people who do not suffer from sleeping disorders or external factors affecting their sleep. The current study demonstrates that a significant proportion of the general population sleeps less than they would like to (see also Soldatos et al., 2005). Corroborating initial findings (Kroese et al., 2014), the current study also shows that experienced sleep insufficiency and actual number of hours of sleep were associated with self-regulation - a relation that was mediated by bedtime procrastination.

Hence, we posit that low self-regulation could be a possible cause for going to bed later than intended. This makes sense considering that people with low self-regulation skills are typically more sensitive to distractions or temptations in the environment, while being less focused on longer-term goals. It can be speculated, for example, that people who have low self-regulation skills are more likely to keep watching the late night movie, or play yet another computer game despite knowing they might regret it the next morning when waking up tired.

However, we hasten to note that, despite the prospective design, it is not possible to make conclusions about the actual causal relationships 


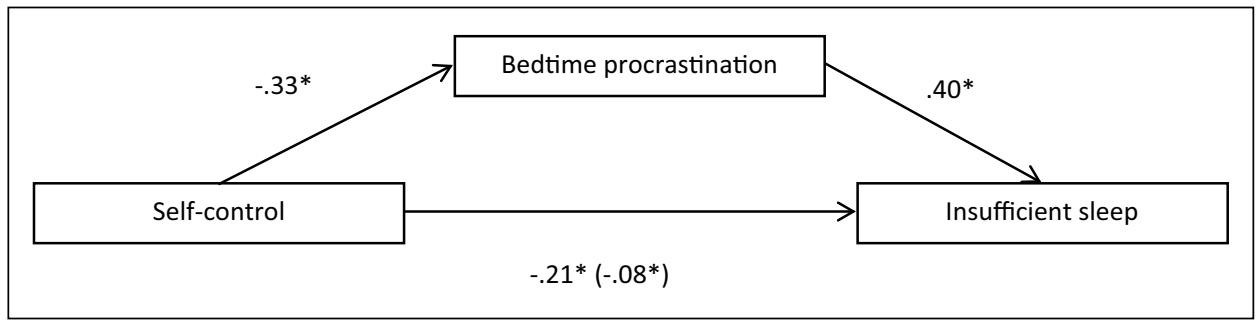

Figure I. Standardized regression coefficients of the relationship between self-control, bedtime procrastination, and experiencing insufficient sleep, controlling for demographics and external reasons.

Table 3. Final regression models for hours of sleep and discrepancy between planned and actual bedtimes.

\begin{tabular}{|c|c|c|c|c|c|c|}
\hline & \multicolumn{3}{|c|}{ Hours of sleep } & \multicolumn{2}{|c|}{ Bedtime discrepancy } & \multirow[t]{2}{*}{$\Delta R^{2}$} \\
\hline & $\beta$ & $p$ & $\Delta R^{2}$ & $\beta$ & $p$ & \\
\hline Step I: & & & .05 & & & .03 \\
\hline Sex ${ }^{a}$ & -.03 & .20 & & -.01 & .76 & \\
\hline Age & -.11 & .00 & & -.12 & .00 & \\
\hline Living w/partnerb & .08 & $<.001$ & & -.08 & .00 & \\
\hline Number of children & -.06 & .02 & & -.01 & .66 & \\
\hline \multicolumn{7}{|l|}{ Employment ${ }^{c}$} \\
\hline Employed & -.13 & $<.001$ & & -.05 & .05 & \\
\hline Student & .11 & $<.001$ & & -.02 & .55 & \\
\hline Step 2: & & & .01 & & & .00 \\
\hline External reasons & -.07 & .00 & & .02 & .32 & \\
\hline Step 3: & & & .01 & & & .01 \\
\hline Self-controld & .01 & .57 & & -.01 & .76 & \\
\hline Step 4: & & & .03 & & & .04 \\
\hline Bedtime procrastination & -.18 & $<.001$ & & .23 & $<.001$ & \\
\hline
\end{tabular}

a $=$ male, 2 = female.

${ }^{\mathrm{b}} 0=$ not living with partner, $\mathrm{I}=$ living with partner.

cemployment was recoded into two dummy variables: employed $(\mathrm{I}=$ employed, $0=$ not employed $)$ and student ( $I$ = student, 0 = not a student).

${ }^{d}$ without bedtime procrastination included in the model, self-control was a significant predictor of both hours of sleep $(\beta=.08, p=.00 \mathrm{I})$ and bedtime discrepancy $(\beta=-.09, p<.00 \mathrm{I})$.

between self-regulation and getting insufficient sleep. In fact, the association between sleep insufficiency and self-regulation has been put forth in a very different way as well. Previous studies have highlighted the consequences of insufficient sleep on self-regulation, showing, for example, that sleep deprived individuals perform worse on self-regulation tasks involving working memory (Gohar et al., 2009) or decision making (Harrison and Horne, 2000).
Importantly, however, we refer to self-regulation as a trait rather than a state and focus on relatively consistent habits of going to bed later than intended. Thus, the current findings suggest that the relation between self-regulation and sleep is not restricted to the earlier views of insufficient sleep leading to temporarily reduced self-regulation. It may well be the case that people who are low in self-regulation as a trait are more likely to go to bed late because they have trouble 
enacting their intentions and/or quitting other activities. Alternatively, there could be a third underlying factor that is associated both with low self-regulation and with insufficient sleep. Therefore, notwithstanding the sensible notion that sleep deprivation may harm (state) self-regulation, the current findings suggest that the relationship between self-regulation and (a lack of) sleep may at least be more complicated than previously acknowledged.

Approaching insufficient sleep from a selfregulation perspective puts it on par with other health behavior problems such as getting too little exercise, or making unhealthy food choices. This has very interesting implications that relate not only to understanding the problem but also to possible solutions. If insufficient sleep is (in part) a self-regulation problem, self-regulation strategies that have proven successful in other behavioral areas could well be applied to this particular case as well. For example, planning strategies such as implementation intentions could be a fruitful remedy against bedtime procrastination.

Strengths of the current study include its prospective design and the large, representative sample of Dutch individuals. This allows for informative descriptive statistics on sleeping behavior in the general population. Notably, unlike much research on insufficient sleep, we did not focus on insomnia or other sleeping disorders. Although insomnia has attracted much more research attention than insufficient sleep in general, it has been noted previously that such factors only account for a minor part of sleep insufficiency in the general population (Broman et al., 1996; Lack et al., 1988).

We particularly encourage further research in the general population that more specifically pinpoints the situations that trigger bedtime procrastination in order to gain insight into relevant situational factors that could be addressed in interventions. For example, just as task aversiveness is known to be an important factor affecting procrastination in academic contexts (Steel, 2007), it might be that bedtime procrastinators experience sleeping or going to bed (e.g. their bedtime routines) as something aversive as well. Furthermore, it is important to further scrutinize the relationship between selfregulation and insufficient sleep: for example, are people with low self-regulation more easily distracted by fun activities in the evening, or is it rather the case that people with low selfregulation have trouble finishing their duties, rendering them doing chores or working late and in this way postponing going to bed?

Finally, we foresee interesting opportunities for future research that investigates possible strategies for helping people go to bed at the time they intended to. Targeting a lack of sleep with self-regulation strategies would be a very novel approach, as most intervention studies so far have focused on, for example, sleep hygiene practices. Moreover, interventions to improve sleeping behavior have not often targeted the general population but have mainly focused on sleep disorder patients. As a first indication that self-regulation interventions could indeed be helpful to improve sleeping behavior, see, for example, the recent work conducted by Loft and Cameron (2013).

In sum, the current study addresses an important but understudied issue by showing the large extent to which people in the general population are experiencing insufficient sleep, and putting this insight into a novel self-regulation perspective. As such, this article may be a stepping stone for further research that will have the opportunity to extend our understanding of the bedtime procrastination phenomenon and investigate possible solutions to help improve normal people's sleeping behavior.

\section{Acknowledgements}

The LISS panel data were collected by CentERdata (Tilburg University, The Netherlands) through its MESS project funded by the Netherlands Organization for Scientific Research. The authors acknowledge Joel Anderson for putting forth the bedtime procrastination concept.

\section{Declaration of conflicting interests}

The author(s) declared no potential conflicts of interest with respect to the research, authorship, and/ or publication of this article. 


\section{Funding}

The author(s) disclosed receipt of the following financial support for the research, authorship, and/or publication of this article: This work was supported by the Dutch Technology Foundation STW, which is part of the Netherlands Organization for Scientific Research (NWO), and which is partly funded by the Ministry of Economic Affairs.

\section{References}

Broman JE, Lundh LG and Hetta J (1996) Insufficient sleep in the general population. Clinical Neurophysiology 26: 30-39.

Buxton OM and Marcelli E (2010) Short and long sleep are positively associated with obesity, diabetes, hypertension, and cardiovascular disease among adults in the United States. Social Science \& Medicine 71: 1027-1036.

De Ridder DTD and De Wit J (2006) Self-regulation of health behavior: Concepts, theories and central issues. In: De Ridder D and De Wit J (eds) Self-Regulation in Health Behavior. Chichester: Wiley, pp. 1-23.

De Ridder DTD, Lensvelt-Mulders G, Finkenauer C, et al. (2012) Taking stock of self-control: A meta-analysis of how trait self-control related to a wide range of behaviors. Personality and Social Psychology Review 16: 76-99.

Gohar A, Adams A, Gertner E, et al. (2009) Working memory capacity is decreased in sleep-deprived internal medicine residents. Journal of Clinical Sleep Medicine 5: 191-197.

Harrison Y and Horne JA (2000) The impact of sleep deprivation on decision making: A review. Journal of Experimental Psychology: Applied 6: 236-249.

Kroese FM, de Ridder DTD, Evers C, et al. (2014) Bedtime procrastination: Introducing a new area of procrastination. Frontiers in Psychology 5: 611.

Lack L, Miller W and Turner D (1988) A survey of sleeping difficulties in an Australian population. Community Health Studies 12: 200-207.

Loft MH and Cameron LD (2013) Using mental imagery to deliver self-regulation techniques to improve sleep behaviors. Annals of Behavioral Medicine 46: 260-272.

National Sleep Foundation (2005) Sleep in America Poll. Washington, DC: National Sleep Foundation.

Ohayon M, Smolensky M and Roth T (2010) Consequences of shift-working on sleep duration, sleepiness and sleep attacks. Chronobiology International 27: 575-589.

Ohida T, Kamal AMM, Uchiyama M, et al. (2001) The influence of life style and health status factors on sleep loss among the Japanese general population. Sleep 24: 333-338.

Ram S, Seirawan H, Kumar SK, et al. (2010) Prevalence and impact of sleep disorders and sleep habits in the United States. Sleep and Breathing 14: 63-70.

Soldatos C, Allaert F, Ohta T, et al. (2005) How do individuals sleep around the world? Results from a single-day survey in ten countries. Sleep Medicine 6: 5-13.

Steel P (2007) The nature of procrastination: A metaanalytic and theoretical review of quintessential self-regulatory failure. Psychological Bulletin 133: 65-94.

Strine T and Chapman D (2005) Associations of frequent sleep insufficiency with health-related quality of life and health behaviors. Sleep Medicine 6: 23-27.

Swanson L, Arnedt J, Rosekind M, et al. (2011) Sleep disorders and work performance: Findings from the 2008 National Sleep Foundation Sleep in America poll. Journal of Sleep Research 20: 487-494.

Tangney JP, Baumeister RF and Boone AL (2004) High self-control predicts good adjustment, less pathology, better grades, and interpersonal success. Journal of Personality 72: 271-324. 


\section{Appendix I}

\section{Bedtime procrastination scale}

For each of the following statements, please decide whether it applies to you using a scale from 1 (almost) never to 5 (almost) always.

1. I go to bed later than I had intended.

2. I go to bed early if I have to get up early in the morning. (reverse coded)

3. If it is time to turn off the lights at night I do it immediately. (reverse coded)

4. Often I am still doing other things when it is time to go to bed.

5. I easily get distracted by things when I actually would like to go to bed.

6. I do not go to bed on time.

7. I have a regular bedtime which I keep to. (reverse coded)

8. I want to go to bed on time but I just do not.

9. I can easily stop with my activities when it is time to go to bed. (reverse coded) 